\title{
No signature of contact ion pairs in the K 2p Auger spectra of highly concentrated potassium halide solutions
}

\author{
Wandared Pokapanich $^{\mathrm{a}, *}$, Niklas Ottosson ${ }^{\mathrm{b}}$, Johan Söderström ${ }^{\mathrm{b}}$, Olle Björneholm ${ }^{\mathrm{b}}$, Gunnar Öhrwall $^{\mathrm{c}}$ \\ a Division of Physics, Faculty of Science, Nakhon Phanom University, 167 Naratchakouy Sub-district, \\ Muang District, Nakhon Phanom 48000 Thailand \\ b Department of Physics and Astronomy, Uppsala University, Box 516, SE-751 20 Uppsala, Sweden \\ c MAX-lab, Lund University, P.O. Box 118, SE-221 00 Lund, Sweden
}

*Corresponding author, e-mail: wandared.pokapanich@npu.ac.th

Received 15 Apr 2013

Accepted 22 Jul 2014

\begin{abstract}
Possible ion pair formation in highly concentrated aqueous potassium halide solutions (fluoride, chloride, and bromide) has been investigated using Auger electron spectroscopy. The potassium Auger spectra exhibit features at high kinetic energy which are related to final states with vacancies on the potassium ion and a neighbouring water molecule. The potassium ion could possibly associate with a halide ion as well, giving rise to additional final states with a vacancy on the halide instead of a water molecule which should give rise to separable spectral features. The necessity of close proximity of the ions for such decays to occur would be strong evidence that contact ions pairs are present in the solution, but no features related to final states involving the anion were observed.
\end{abstract}

KEYWORDS: aqueous solution, liquid micro-jet, double ionization potential, localized state

\section{INTRODUCTION}

Ion pairing plays an important role in aqueous electrolytic solutions and is found in various forms. A possible dyadic situation is the contact ion pair (CIP) in which the constituent ions are in direct contact and not separated by other neutral molecules such as an intervening solvent water molecule in the case of aqueous solutions. If the ions are separated by only a single solvent molecule, the situation is designated as a solvent-shared ion pair (SIP) ${ }^{1,2}$.

The prevalence and characteristics of ion pairs have been studied using various methods, both experimental and computational. Max et al used attenuated total reflection infrared spectroscopy to characterize aqueous salt solutions close to saturation (e.g., $4.03 \mathrm{M}$ $\mathrm{KCl}$ and 4.20 M KBr). They found that at these concentrations, the anion and cation form an aqueous bonded pair, associated with a number of neighbouring water molecules, which is dependent on the ratio of the ionic radius of the ions ${ }^{3,4}$. For two of the ions studied in the present paper, $\mathrm{KCl}$ and $\mathrm{KBr}$, the anion-to-cation ionic radius ratios are 1.36 and 1.47 , respectively, and the number of associated water molecules that was found for these salts was close to 5 which agreed with the ab initio calculation from $\mathrm{Sen}^{5}$. For KF, which was not part of their study, the ratio is close to 1.0 which, according to their model, would indicate a smaller number of associated water molecules. Although they claim that the ions are closely bound in a complex, the method relies on analysis of water spectra and does not reveal if the ions are in immediate contact.

Fennell and co-workers have used molecular dynamics simulations to investigate CIPs and SIPs in low concentration alkali halide solutions ${ }^{6}$. The simulations showed that the probability of forming CIPs and SIPs correlated with cation and anion sizes; smallsmall and large-large should associate in water. When both ions are of similar size, particularly when both ions are small, as for $\mathrm{LiF}$, there exist potential minima for the formation of CIPs, whereas when the two ionic radii are different, as for LiI, SIPs would be more likely. This correlates well with the 'law of matching water affinities model' where the relative affinity of the ions depends on how close the cation and anion sizes are ${ }^{7,8}$. Judging from the simulations of Fennel and co-workers, the potassium salts investigated in this paper would not be the most predisposed for CIP formation among the alkali halides, but they indicate that CIPs would be least likely for $\mathrm{KF}^{6}$. However, for a near-saturated aqueous solution of KF $(16.15 \mathrm{~m})$, Ohtaki and Fukushima using X-ray diffraction reached the conclusion that the average $\mathrm{K}^{+}-\mathrm{F}^{-}$coordination number is 2.3 , suggesting that CIPs are common. For a $4.56 \mathrm{~m}$ aqueous solution 
of $\mathrm{KCl}$, they found the average $\mathrm{K}^{+}-\mathrm{Cl}^{-}$coordination number is 0.6 , indicating the presence of some CIPs $^{9}$. Benjamin also investigated ion pairs in $\mathrm{KCl}$, $\mathrm{KI}$, and KF surrounded by two water molecules by using molecular dynamics computer simulations ${ }^{10}$. The author found that the ions remain in contact for aqueous $\mathrm{KCl}$ and $\mathrm{KI}$, but not in $\mathrm{KF}$.

Auger electron spectroscopy (AES) is a powerful technique for investigating the electronic structure of matter. It utilizes the deep lying core levels, which are localized and therefore give atom-specific and local information, to probe the valence levels which are sensitive to the surroundings. Recently, electron spectroscopy has become more accessible for investigations of liquids and solutions through the development of the liquid micro-jet technique ${ }^{11}$. In this work, we have used this technique combined with AES to characterize highly concentrated aqueous $\mathrm{KF}$, $\mathrm{KCl}$, and $\mathrm{KBr}$, especially with respect to the question of the presence of CIPs in these solutions.

In an earlier paper, we investigated aqueous solutions of $\mathrm{KCl}$, and we observed Auger final states involving vacancies on neighbouring centres to the $\mathrm{K}^{+}$ion after ionization of the $\mathrm{L}_{2,3}$ edges ${ }^{12}$. Highlevel ab initio calculations on model clusters showed that these were most probably due to water molecules in the first solvation shell. What is clear from the theoretical modelling is that only centres in the immediate vicinity of the core-ionized atom take part in such intermolecular decay channels, meaning solventshared ion pairs would not be expected to contribute to the Auger spectrum. To utilize this sensitivity to the immediate surroundings of the potassium ions, we decided to systematically exchange the counter ion, since the involvement of the anion in the Auger decay would change the final state energies and would be observable in the spectra. Changes in the features related to the intermolecular decays between the solutions would thus be clear evidence of CIPs, and the opposite situation would indicate the absence of CIPs.

\section{EXPERIMENTAL DETAILS}

Aqueous solutions of $\mathrm{KF}, \mathrm{KCl}$, and $\mathrm{KBr}$ were prepared by mixing high purity salts $(\geqslant 99 \%$, Sigma Aldrich) with deionized water to obtain a concentration of 4 molal (m). At these concentrations, nearly all water molecules will be in the solvation shell of a cation $\left(\mathrm{K}^{+}\right)$or an anion $\left(\mathrm{F}^{-}, \mathrm{Cl}^{-}\right.$, and $\left.\mathrm{Br}^{-}\right)$. The experiments were performed at the Swedish synchrotron radiation facility MAX-lab at the undulator-based soft $\mathrm{X}$-ray beamline $\mathrm{I} 411^{13,14}$ equipped with a Scienta R4000 electron spectrometer mounted at a magic angle of $54.7^{\circ}$ in order to minimize angular effects.
The spectra were obtained using a $15 \mu \mathrm{m}$ liquid jet injected into a differentially-pumped vacuum chamber. The jet was perpendicular to the direction of the photon beam and the spectrometer. Typical working pressures were in the $10^{-5}$ mbar range in the differential pumping stage and in the $10^{-6}$ mbar range in the analyser chamber. The experimental setup is described in more detail in Ref. 15.

The $\mathrm{K}^{+} 2 \mathrm{p}$ Auger electron spectra were recorded with two different photon energies to avoid spectral overlap of the photoline and loss features from the $\mathrm{Br} 3 \mathrm{~d}$ core level for the $\mathrm{KBr}$ solution. We used aqueous $\mathrm{KCl}$ as the reference for both aqueous $\mathrm{KF}$ and $\mathrm{KBr}$. Spectra for aqueous $\mathrm{KF}$ and $\mathrm{KCl}$ were recorded with the photon energy $340 \mathrm{eV}$. Then spectra for solutions of $\mathrm{KCl}$ and $\mathrm{KBr}$ were recorded using the photon energy $385 \mathrm{eV}$. In addition, a spectrum in the kinetic energy range of $\mathrm{K}^{+} 2 \mathrm{p}$ Auger for aqueous $4.0 \mathrm{~m} \mathrm{NaBr}$ was recorded with the same photon energy as the $\mathrm{KBr}$ solution to ensure that no bromide feature is influencing the spectrum.

\section{RESULTS AND DISCUSSION}

We will begin the description with the main potassium $\mathrm{L}_{2,3} \mathrm{M}_{2,3} \mathrm{M}_{2,3}$ Auger features which correspond to kinetic energies between 235 and $255 \mathrm{eV}$ (Fig. 1). The spectra in this region are very similar for the different aqueous potassium halides. The spectra are similar to that of solid $\mathrm{KCl}$, but rather dissimilar to those of gas phase $\mathrm{KCl}, \mathrm{KBr}$, and $\mathrm{KI}^{16,17}$. It has been shown that this dissimilarity between potassium halide molecules and solids can be be explained by strong interactions in the final state in the molecules which are not present in the crystals due to their high symmetry ${ }^{17}$. We learned from Ref. 12 that for the solvated ion, the spectra can essentially be interpreted using a raregas (argon) picture, with $\mathrm{K}^{+3} 3 \mathrm{p}^{-2}$ localized final states $\left({ }^{3} \mathrm{P},{ }^{1} \mathrm{D}\right.$, and $\left.{ }^{1} \mathrm{~S}\right)$. There is a slight difference between the spectra recorded at 340 and $385 \mathrm{eV}$ in the relative intensities of the peaks. This may be due to a photon energy dependence of the branching ratio of the spin-orbit components, as has been observed in free potassium halide molecules ${ }^{17}$.

The most interesting feature is the weak structure that lies between 255 and $270 \mathrm{eV}$, i.e., at higher energies than the main feature. The higher kinetic energy of the electrons means that the final state has lower energy than the lowest possible $3 \mathrm{p}^{-2}$ states. As shown in Ref. 12, this feature is due to delocalized Auger final states with one vacancy in the $3 p$ shell of the core-ionized potassium ion and one valence hole on a neighbouring site, $3 \mathrm{p}^{-1} \mathrm{~V}^{-1}$, where $\mathrm{V}$ stands for a water or anion valence level. Such states have 


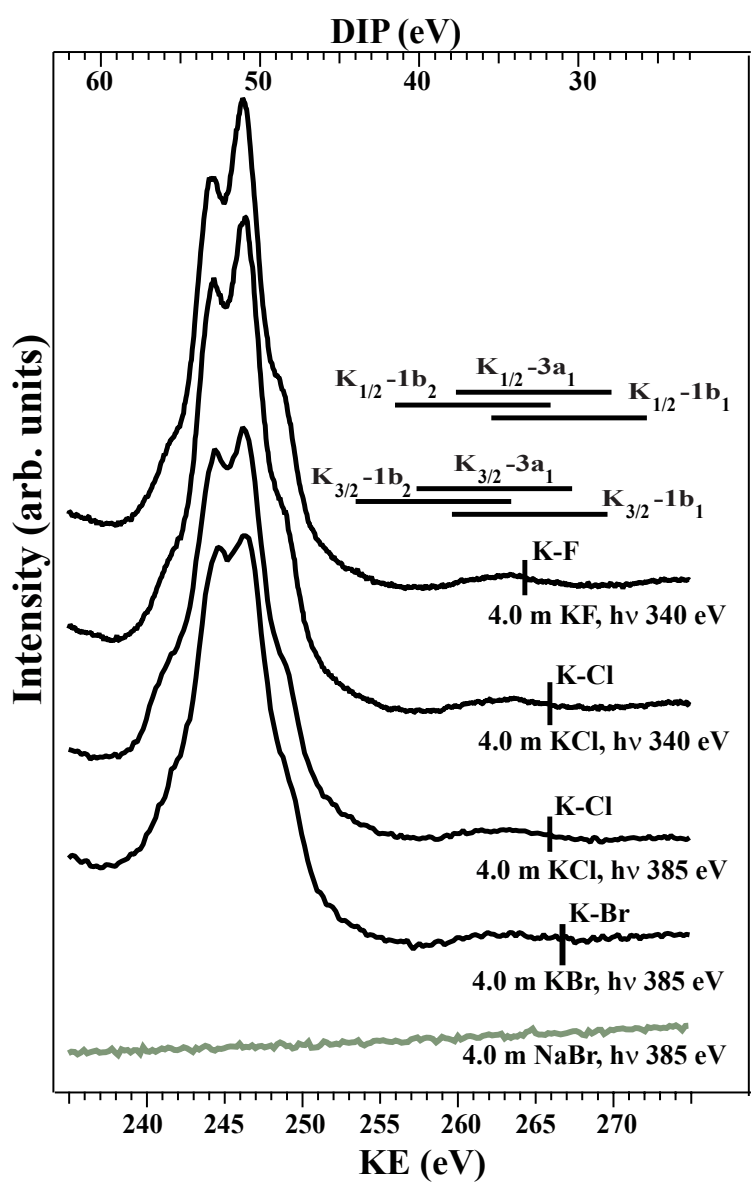

Fig. 1 Auger spectra of $\mathrm{K}^{+}$after $2 \mathrm{p}$ photoionization in aqueous $\mathrm{KF}, \mathrm{KBr}$, and $\mathrm{KCl}$. The photon energies used were $340 \mathrm{eV}$ (KF: top; $\mathrm{KCl}$ : second from top) and $385 \mathrm{eV}$ ( $\mathrm{KCl}$ : third from top; $\mathrm{KBr}$ : fourth from top). The bottom spectrum comes from an aqueous $4.0 \mathrm{~m} \mathrm{NaBr}$ solution and is used to verify that loss features from $\mathrm{Br} 3 \mathrm{~d}$ in the $\mathrm{KBr}$ spectrum feature are negligible. Estimated ranges for double ionizations potentials (DIP) of delocalized final states involving a vacancy on a water molecules, denoted $\mathrm{K}-1 \mathrm{~b}_{1}, \mathrm{~K}-3 \mathrm{a}_{1}$, and $\mathrm{K}-1 \mathrm{~b}_{2}$, are shown as horizontal lines. Subscripts $K_{1 / 2}$ and $K_{3 / 2}$ represent $K 2 p_{1 / 2}$ and $K 2 p_{3 / 2}$, respectively. The DIP of final states involving the anions are shown as vertical bars.

lower energy than the $3 \mathrm{p}^{-2}$ states mainly because of the reduced Coulomb repulsion. The calculations of the Auger spectra in Ref. 12 were performed for $\mathrm{KCl}\left(\mathrm{H}_{2} \mathrm{O}\right)_{1-6}$ model clusters. For clusters containing a small number of water molecules, such as $\mathrm{KCl}\left(\mathrm{H}_{2} \mathrm{O}\right)_{1-3}$, contributions from states with a $\mathrm{Cl}^{-}$ valence vacancy can clearly be seen in the spectrum at a higher kinetic energy than the corresponding water vacancy final states. The intensity of the delocalized states involving a $\mathrm{Cl}^{-}$vacancy decreases gradually with hydration, and is negligible for a hexahydrated $\mathrm{KCl}$ cluster where delocalized states with a water vacancy dominate. The method is thus sensitive to ions in the immediate surroundings and AES could therefore be a suitable tool to investigate issues related to this.

In Fig. 1, we have included estimates of energy ranges for the double ionization potentials (DIP) of the delocalized final states. The horizontal bars show intervals for states involving holes in valence orbitals on a neighbouring water molecule: $3 \mathrm{p}^{-1}\left(1 \mathrm{~b}_{1}\right)^{-1}$, $3 \mathrm{p}^{-1}\left(3 \mathrm{a}_{1}\right)^{-1}$, and $3 \mathrm{p}^{-1}\left(1 \mathrm{~b}_{2}\right)^{-1}$, denoted $\mathrm{K}-1 \mathrm{~b}_{1}$, K$3 \mathrm{a}_{1}$, and $\mathrm{K}-1 \mathrm{~b}_{2}$, respectively. These are shown for decays from both $\mathrm{K} 2 \mathrm{p}$ spin-orbit components, but the scale at the top of the figure is for a $\mathrm{K} 2 \mathrm{p}_{3 / 2}$ intermediate state. The energies were simply estimated as the sum of the vertical binding energies of the respective single hole states plus a Coulomb term, accounting for the Coulomb interaction in the two-hole final state. The upper bound of the range corresponds to the estimated DIP including an unscreened Coulomb interaction term between the $\mathrm{K}^{2+}$ and the $\left(\mathrm{H}_{2} \mathrm{O}\right)^{+}$ ions, and the lower bound corresponds to a completely screened case, where there is no Coulomb interaction between the ions. Additionally, vertical lines indicate estimated DIPs for Auger final states with a vacancy in the outermost p-orbital of the anion for a decay from the $2 p_{3 / 2}$ core-ionized intermediate state, which we have denoted $\mathrm{K}-\mathrm{F}, \mathrm{K}-\mathrm{Cl}$, and $\mathrm{K}-\mathrm{Br}$. These could be observed in the case of prevalent contact ion pairs. Note that there will be no Coulomb interaction in the final state, as the anion will become neutral upon photodetachment.

The single ionization energies used for the estimate of the DIP of the final state in the Auger spectra were derived from several sources. The liquid water $1 b_{1}$-state, $3 a_{1}$-state, and $1 b_{2}$-state are at $11.16,13.50$, and $17.34 \mathrm{eV}$, respectively ${ }^{18}$. The core photoelectron binding energies of the $\mathrm{K}^{+} 2 \mathrm{p}$ level are 303.55 and $297.74 \mathrm{eV}$ for the $\mathrm{K}^{+} 2 \mathrm{p}_{1 / 2}$ and $\mathrm{K}^{+} 2 \mathrm{p}_{3 / 2}$ components, respectively ${ }^{12}$. The valence binding energies of the aqueous ion levels $\mathrm{K}^{+} 3 \mathrm{p}, \mathrm{Br}^{-} 4 \mathrm{p}$, and $\mathrm{Cl}^{-} 3 \mathrm{p}$ are at $22.2,8.8$, and $9.6 \mathrm{eV}$, respectively ${ }^{12,19}$. The $\mathrm{F}^{-}$ $2 p$ line strongly overlaps with the water $1 b_{1}$ emission peak $^{20}$, and for this reason an accurate value of the outermost valence binding energy of $\mathrm{F}^{-} 2 \mathrm{p}$ has not been reported in the aqueous phase. In this work we have estimated the $\mathrm{F}^{-}$valence energy approximately as that of the water $\mathrm{X}$-state $(11 \mathrm{eV})$. We used literature values for the distance of $\mathrm{K}^{+}$-water in aqueous $\mathrm{KF}, \mathrm{KCl}$, and $\mathrm{KI}^{9,21,22}$, to estimate the maximum Coulomb repulsion. The adjacent $\mathrm{K}^{+}$-water distances 
are between 2.8 and $2.9 \AA$, resulting in a Coulomb repulsive term of 9.9-10.3 eV. The average minimum DIP (maximum in parentheses) for the states $\mathrm{K}-1 \mathrm{~b}_{1}$, $\mathrm{K}-3 \mathrm{a}_{1}$, and $\mathrm{K}-1 \mathrm{~b}_{2}$ are 33.4 (43.5), 35.7 (45.8), and 39.5 (49.6) eV, respectively.

The final states with one hole on potassium and one hole on water fit the observed feature for the case where the final state ions are efficiently screened from each other. This was also borne out by the ab initio calculations presented in Ref. 12. It is thus clear that these features are at least partly due to the Auger decays from the $\mathrm{K}^{+2} 2 \mathrm{p}^{-1}$ state to a $\mathrm{K}^{+3} 3 \mathrm{p}^{-1}$ and a water valence vacancy state. However, there is still the question of whether only such states are populated, or if CIP states contribute.

In the calculation of the energies of final states with a vacancy on an anion, there is no Coulomb term to consider in the final state since the anion becomes neutral upon photodetachment. However, there could possibly be a Coulombic contribution in the coreionized intermediate state. This should appear in the photoelectron spectrum as a feature chemically shifted from the line for an ion which is only coordinated to neutral water molecules. We have not observed any such chemically shifted $\mathrm{K} 2 \mathrm{p}$ line at any concentration for these potassium salts. This either means that the Coulomb interaction is well screened or that there are no CIPs. We have therefore estimated the DIP energy for the possible CIP as the sum of the single hole energies: $33.4,31.8$, and $31.0 \mathrm{eV}$ for $\mathrm{K}-\mathrm{F}, \mathrm{K}-\mathrm{Cl}$, and $\mathrm{K}-\mathrm{Br}$, respectively. In the case of the $\mathrm{Cl}^{-}$and $\mathrm{Br}^{-}$ counter ions, these energies are clearly lower than the lowest energies for states with water vacancies.

By subtracting the spectra from each other, possible minute differences will appear clearer, and we have done so to investigate the possible presence of CIP final states in the $\mathrm{K} 2 \mathrm{p}$ Auger spectra in the structures at high kinetic energy $255-270 \mathrm{eV}$ or DIP 28 $43 \mathrm{eV}$. The difference spectra of aqueous KF minus $\mathrm{KCl}$ (red solid line, top) and aqueous $\mathrm{KBr}$ minus $\mathrm{KCl}$ (black solid line, bottom) are shown in Fig. 2. The spectra show no structure in the region where such final states would appear, which suggests that CIPs are not common for these potassium salts at the studied concentration.

Using the empirical 'law of matching water affinities' Collins et al suggested that for aqueous potassium halide solutions, the probability of forming the CIPs $\mathrm{K}^{+}-\mathrm{Br}^{-}$and $\mathrm{K}^{+}-\mathrm{Cl}^{-}$is higher than $\mathrm{K}^{+}-\mathrm{F}^{-7,8}$. The explanation is that the $\mathrm{K}^{+}, \mathrm{Br}^{-}$, and $\mathrm{Cl}^{-}$are weakly hydrated (large size, low surface charge density), and were designated as chaotropes. When the crystalline chaotrope-chaotrope alkali halides $(\mathrm{KBr}$

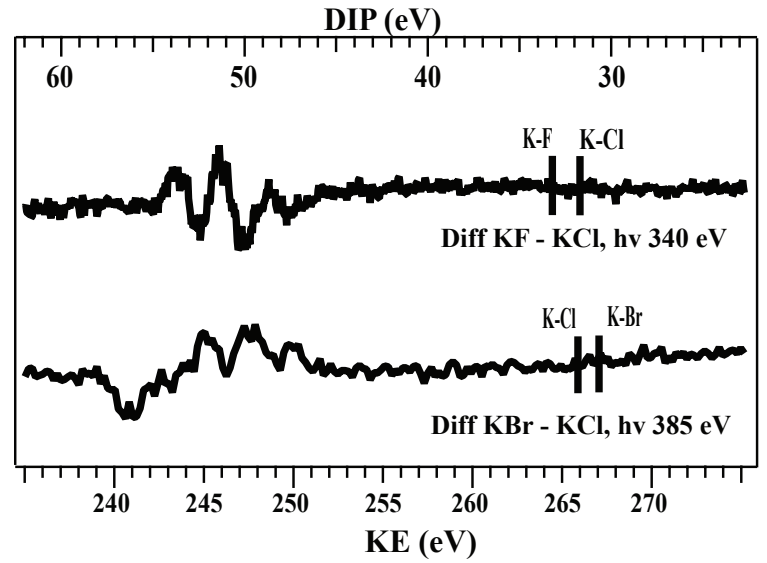

Fig. 2 Difference spectra of aqueous $\mathrm{KF}$ minus $\mathrm{KCl}$ and aqueous $\mathrm{KBr}$ minus $\mathrm{KCl}$, recorded at the same photon energies, represented by the top and bottom solid lines, respectively. The possible CIP energies are indicated by the vertical bars.

and $\mathrm{KCl}$ ) dissolve in water, relatively strong waterwater interactions will keep the chaotrope ion pairs together. When the constituent ions do dissociate, relatively strong water-water interactions will be broken. In contrast, the $\mathrm{F}^{-}$ion was designated as a kosmotrope, being strongly hydrated (small size, high surface charge density). Aqueous KF is then a chaotrope-kosmotrope compound, where the kosmotropic ion of this salt interacts more strongly with water in solution than with the chaotrope in the crystal. Consequently, both the solvated $\mathrm{K}^{+}$and $\mathrm{F}^{-}$ions will be preferentially attached to water neighbours. These considerations correlate well with the solubility of these salts. In our study, the concentrations of the $\mathrm{KCl}$ and $\mathrm{KBr}$ solutions are closer to saturation than the $\mathrm{KF}$ solution, but our method indicates that even for this situation, few CIPs are formed.

\section{CONCLUSIONS}

We have investigated $4.0 \mathrm{~m}$ aqueous solutions of $\mathrm{KF}$, $\mathrm{KCl}$, and $\mathrm{KBr}$ using AES to explore the presence of contact ion pairs at this concentration. From our previous study of $\mathrm{KCl}$, in the potassium $2 \mathrm{p}$ Auger spectrum, final states involving holes on neighbouring centres are present, and ab initio calculations showed that these predominantly come from water vacancies, but that anion contributions should be expected if the anion is in the direct vicinity of the cation. Here, we have compared the $\mathrm{KCl}$ spectrum to spectra for $\mathrm{KF}$ and $\mathrm{KBr}$, and no significant difference is found in the region where final states from the anions can be expected. This suggests that CIPs are uncommon at this 
concentration. This finding is somewhat surprising as the $\mathrm{KCl}$ and $\mathrm{KBr}$ solutions are not far from saturation when precipitating salt molecules clearly have to be in close vicinity.

Auger spectroscopy is an emerging tool for studies of solutions, and this study could easily be extended to other electrolytic solutions, showing the power of this method.

Acknowledgements: This work has been financially supported by the Swedish Research Council (VR), the Göran Gustafsson foundation, the Knut and Alice Wallenberg foundation, and the Carl Tryggers Foundation. We thank Nikolai V. Kryzhevoi for fruitful discussions. We also thank the MAX-lab staff for their assistance during the experiments.

\section{REFERENCES}

1. Plodinec J, Hogen-Esch TE (1974) Ion pairing in excited states of carbanions. I. Cation and solvent effects. J Am Chem Soc 96, 5262-4.

2. Antonio MR, Nyman M, Anderson TM (2009) Direct observation of contact ion-pair formation in aqueous solution. Angew Chem Int Ed 48, 6136-40.

3. Max JJ, Chapados C (2001) IR spectroscopy of aqueous alkali halide solutions: Pure salt-solvated water spectra and hydration numbers. J Chem Phys 115, 2664-75.

4. Max JJ, Chapados C (2007) Infrared spectroscopy of aqueous ionic salt mixtures at low concentrations: Ion pairing in water. J Chem Phys 127, 114509.

5. Sen A, Ganguly B (2010) What is the minimum number of water molecules required to dissolve a potassium chloride molecule? J Comput Chem 31, 2948-54.

6. Fennell CJ, Bizjak A, Vlachy V, Dill KA (2009) Ion pairing in molecular simulations of aqueous alkali halide solutions. J Phys Chem B 113, 6782-91.

7. Collins KD, Neilson GW, Enderby JE (2007) Ions in water: Characterizing the forces that control chemical processes and biological structure. Biophys Chem 128, 95-104.

8. Collins KD (1997) Charge density-dependent strength of hydration and biological structure. Biophys $J \mathbf{7 2}$, 65-76.

9. Ohtaki H, Fukushima N (1992) A structural study of saturated aqueous solutions of some alkali halides by X-ray diffraction. J Solution Chem 21, 23-38.

10. Benjamin I (2010) Structure and dynamics of hydrated ion pairs in a hydrophobic environment. J Phys Chem B 114, 13358-64.

11. Winter B, Faubel M (2006) Photoemission from liquid aqueous solutions. Chem Rev 106, 1176-211.

12. Pokapanich W, Bergersen H, Bradeanu IL, Marinho R, Lindblad A, Legendre S, Rosso A, Svensson S, et al (2009) Auger electron spectroscopy as a probe of the solution of aqueous ions. J Am Chem Soc 131, 7264-71.

13. Bässler M, Forsell JO, Björneholm O, Feifel R, Jurvansuu M, Aksela S, Sundin S, Sorensen SL, et al (1999) Soft X-ray undulator beam line I411 at MAX II for gases, liquids and solid samples. J Electron Spectros Relat Phenom 101-103, 953-7.

14. Bässler M, Ausmees A, Jurvansuu M, Feifel R, Forsell JO, Fonseca PT, Kivimäki A, Sundin S, et al (2001) Beam line I411 at MAX II-performance and first results. Nucl Instrum Meth Phys Res A 469, 382-93.

15. Bergersen H, Marinho RRT, Pokapanich W, Lindblad A, Björneholm O, Sæthre LJ, Öhrwall G (2007) A photoelectron spectroscopic study of aqueous tetrabutylammonium iodide. J Phys Condens Matter 19, 326101.

16. Kukk E, Huttula M, Aksela H, Aksela S, Nõmmiste E, Kikas A (2003) Limitations of the ionic model in describing core-hole decay-molecular versus crystalline KCl. J Phys B 36, L85-91.

17. Patanen $M$, Niskanen J, Huttula M, Jänkälä K, Urpelainen S, Aksela H, Aksela S (2008) Strong molecular field effects in auger decay of the potassium $2 \mathrm{p}$ core-hole state in molecular $\mathrm{KCl}, \mathrm{KBr}$ and KI. $J$ Phys B 41, 215103.

18. Winter B, Weber R, Widdra W, Dittmar M, Faubeland M, Hertel IV (2004) Full valence band photoemission from liquid water using EUV synchrotron radiation. J Phys Chem A 108, 2625-32.

19. Winter B, Weber R, Hertel IV, Faubel M, Jungwirth P, Brown EC, Bradforth SE (2005) Electron binding energies of aqueous alkali and halide ions: EUV photoelectron spectroscopy of liquid solutions and combined $\mathrm{ab}$ initio and molecular dynamics calculations. $J \mathrm{Am}$ Chem Soc 127, 7203-14.

20. Weber R, Winter B, Schmidt PM, Widdra W, Hertel IV, Dittmar M, Faubeland M (2004) Photoemission from aqueous alkali-metal-iodide salt solutions using EUV synchrotron radiation. J Phys Chem B 108, 4729-36.

21. Ohtaki H (2001) Ionic solvation in aqueous and nonaqueous solutions. Monatsh Chem Chem Mon 132, 1237-68.

22. Fishkis MY, Soboleva TE (1974) X-ray structural investigation of aqueous solutions of $\mathrm{KI}$ and LiI. $\mathrm{Zh}$ Strukt Khim 15, 175-80. 